\title{
Nonlinear time series analysis of the human electrocardiogram
}

\author{
Matjaž Perc \\ Department of Physics, Faculty of Education, University of Maribor, Koroška cesta 160, \\ SI-2000 Maribor, Slovenia \\ E-mail: matjaz.perc@guest.arnes.si
}

Received 10 March 2005, in final form 2 May 2005

Published 21 June 2005

Online at stacks.iop.org/EJP/26/757

\begin{abstract}
We analyse the human electrocardiogram with simple nonlinear time series analysis methods that are appropriate for graduate as well as undergraduate courses. In particular, attention is devoted to the notions of determinism and stationarity in physiological data. We emphasize that methods of nonlinear time series analysis can be successfully applied only if the studied data set originates from a deterministic stationary system. After positively establishing the presence of determinism and stationarity in the studied electrocardiogram, we calculate the maximal Lyapunov exponent, thus providing interesting insights into the dynamics of the human heart. Moreover, to facilitate interest and enable the integration of nonlinear time series analysis methods into the curriculum at an early stage of the educational process, we also provide user-friendly programs for each implemented method.
\end{abstract}

\section{Introduction}

Four decades ago, the paradigm of classical time-continuous chaos was introduced [1]. Since the discovery of chaos, the interest in this field of research has risen rapidly and several attempts have been made to integrate this fascinating topic into graduate as well as undergraduate curricula [2-15]. Still, however, insights into the experimentally observed irregular behaviour of systems are predominantly obtained solely with computer simulations of appropriate mathematical models. This approach often works against the student's initial enthusiasm and interest. Moreover, it undermines the trustworthiness of the whole chaos paradigm since it leads to suspect that chaos is nothing more than a mathematical artefact, a phenomenon non-existing outside the simulations of the computer. The remedy to this problem lies in the integration of nonlinear time series analysis methods into the physics curriculum at an early stage of the educational process. While there exist excellent monographs on nonlinear time series analysis [16-18], there is still a shortage of literature showing concrete applications 
of simple methods to real-life problems [15]. This paper is aimed to rectify the situation by showing concrete applications of simple nonlinear time series analysis methods on the human electrocardiogram.

Nonlinear time series analysis is a powerful theory that enables the extraction of characteristic quantities, e.g. the number of active degrees of freedom or invariants, such as the maximal Lyapunov exponent, of a particular system solely by analysing the time course of one of its variables. Thus, the theory of nonlinear time series analysis offers tools that bridge the gap between experimentally observed irregular behaviour and deterministic chaos theory [19-22]. Although, in this sense, the transition between deterministic chaos theory and the analysis of irregular experimental traces appears smooth, the reality is very different. The problem lies in the origin of methods for nonlinear time series analysis, which is the theory of deterministic dynamical systems. Thus, if we are to successfully apply methods of nonlinear time series analysis to experimental data, we first have to verify if the data possess properties typical of deterministic systems. Moreover, we have to verify if the observed irregular behaviour originates from a stationary system, for it might solely be a consequence of varying system parameters during data acquisition. These are very important issues that have to be addressed before attempting further analyses, as we will exemplify in the next section.

We start the study by introducing the embedding theorem [23, 24], which enables the reconstruction of the phase space from a single observed variable, thereby laying foundations for further analyses. Since all experimentally obtained data are more or less contaminated by noise, we then implement a simple noise reduction algorithm [25] to extract the electrocardiographic signal from the time series. Thereby, we assume that the studied time series consists of two components, i.e. the signal and random fluctuations, which have to be separated. After extracting the 'clean' electrocardiographic signal from the experimental data, we use the mutual information [26] and false nearest neighbour method [27] to obtain optimal embedding parameters for the phase space reconstruction. As a prelude to the application of a classical determinism [28] and stationarity [29] test, we apply the method of recurrence plots [30]. The recurrence plot is a powerful graphical tool enabling the assessment of determinism [31] and stationarity [32] in the system, as well as execution of other tasks, such as for example the estimation of the noise level in a signal [33] or the evaluation of correctness of chosen embedding parameters for the phase space reconstruction [31, 34]. Next, we apply the determinism [28] and stationarity [29] test to quantify visually assessed results obtained with the recurrence plot analysis. Note that deterministic chaos is only one possible source of complex irregular behaviour. Other sources, for example, are noise or varying system parameters during data acquisition. By applying the determinism test we are able to determine whether the analysed irregular behaviour is indeed a consequence of deterministic dynamics, while the stationarity test enables us to verify if system parameters were held constant during data recording. After establishing that the studied electrocardiogram originates from a deterministic stationary system, we calculate the maximal Lyapunov exponent [35]. We find that the latter is positive, from which we conclude that the studied human electrocardiographic recording possesses properties typical of deterministic chaotic signals.

Due to the existence of excellent monographs on nonlinear time series analysis [16-18], we do not describe algorithms of implemented methods in great detail. We do, however, provide user-friendly programs with graphical interface [36], which should make the reproduction of presented results possible even for individuals with little or no experience with nonlinear time series analysis and also facilitate further applications of the described methods to other experimental systems. A detailed manual for the set of programs can be found at the download site [36]. 


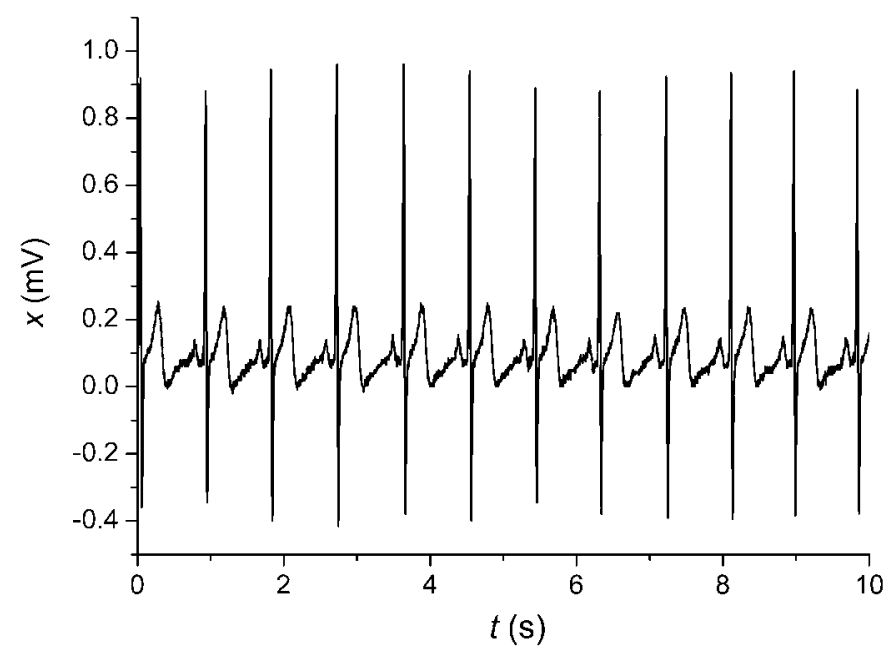

Figure 1. The studied human electrocardiogram.

\section{Results}

We analyse a short, densely sampled electrocardiographic recording of the human heart, which was obtained from the publicly accessible MIT Polysomnographic database [37]. Although up to $6 \mathrm{~h}$ long recordings are available, we use only a short insert from the file slp01a.dat, during which the subject was normally asleep without any significant movement or apnoea attacks. The studied time series consists of 45000 data points and was sampled at $\mathrm{d} t=0.004 \mathrm{~s}$. Thus, a total of $180 \mathrm{~s}$ of electrochemical heart activity is available for study, from which the first $10 \mathrm{~s}$ are shown in figure 1 . It can be observed that the electrocardiogram shows normal and rather regular heart activity with a predominant beat rate of $\approx 66$ beats $\mathrm{min}^{-1}$. Moreover, it can be inferred that the electrocardiogram is quite heavily burdened with noise, especially in between successive peaks.

Following the succession of tasks we have outlined in the introduction, let us start the study by introducing the embedding theorem [23, 24]. The embedding theorem states that for a large enough embedding dimension $m$, the delay vectors

$$
\mathbf{p}(i)=\left(x_{i}, x_{i+\tau}, x_{i+2 \tau}, \ldots, x_{i+(m-1) \tau}\right)
$$

yield a phase space that has exactly the same properties as the one formed by the original variables of the system. In equation (1), variables $x_{i}, x_{i+\tau}, x_{i+2 \tau}, \ldots, x_{i+(m-1) \tau}$ denote values of the electrocardiographic signal at times $t=i \mathrm{~d} t, t=(i+\tau) \mathrm{d} t, t=(i+2 \tau) \mathrm{d} t, \ldots$, $t=(i+(m-1) \tau) \mathrm{d} t$, respectively, where $\tau$ is the so-called embedding delay.

Although the implementation of equation (1) is straightforward, we first have to determine proper values for embedding parameters $\tau$ and $m$. For this purpose, the mutual information [26] and false nearest neighbour method [27] can be used. However, since the examined time series is quite heavily burdened with noise (see figure 1), all further analyses would yield dubious results. Hence, we first have to use a simple noise reduction method to extract the 'clean' electrocardiographic signal from the examined data set. According to Schreiber [25], the noise level in an examined time series can be reduced simply by replacing the middle coordinate, i.e. $x_{i+(m / 2) \tau}$ for even or $x_{i+((m+1) / 2) \tau}$ for odd $m$, of each embedding vector $\mathbf{p}(i)$ by the average value of the middle coordinate obtained from all those embedding vectors $\mathbf{p}(k)$ 


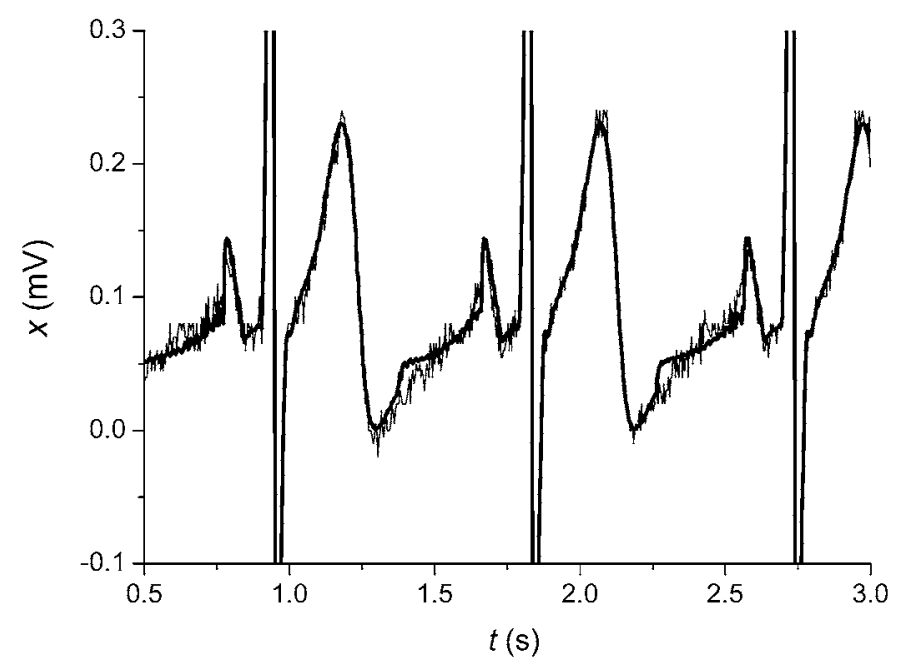

Figure 2. Magnified part of the human electrocardiogram before (thin line) and after (thick line) the noise reduction. Note that the noise amplitude has decreased substantially, while the curvature radius of the signal was largely preserved. See also figure 4 for a $2 \mathrm{D}$ phase space view. This result can be reproduced with the program noisered.exe [36].

that are closer to $\mathbf{p}(i)$ than some chosen $\varepsilon$. For more details and a precise algorithm description we refer the reader to p 51 in [17]. Obviously, the method requires three input parameters, which are $\tau, m$ and $\varepsilon$. The results presented in figure 2 were obtained with $\tau=5, m=15$ and $\varepsilon=0.065$. For noise reduction methods, larger $m$ are preferable for two reasons. First, if any deterministic signature is present in the data set it will be better expressed for large $m$, and second, the selectivity for appropriate neighbours $(\mathbf{p}(k))$ increases with increasing $m$. In alliance with the chosen $m, \tau$ should be chosen such that $m \mathrm{~d} t \tau$ ranges from $1 / 3$ to $2 / 3$ of the predominant oscillation period of the signal. In our case $m \mathrm{~d} t \tau=0.3 \mathrm{~s}$, which is approximately $1 / 3$ of the average beat interval. Finally, $\varepsilon$ should be larger than the noise amplitude, but still small enough not to average out the typical curvature radius of the time series. Normally, a careful visual inspection of the time series and a few test runs are necessary to obtain the optimal result.

Next, we apply the mutual information [26] and false nearest neighbour method [27] to the newly obtained 'clean' electrocardiographic signal to obtain proper embedding parameters. The mutual information between $x_{i}$ and $x_{i+\tau}$ can be used to estimate a proper embedding delay $\tau$. A suitable $\tau$ has to fulfil two criteria. First, $\tau$ has to be large enough so that the information we get from measuring the value of variable $x$ at time $i+\tau$ is relevant and significantly different from the information we already have by knowing the value at time $i$. Only then will it be possible to gather enough information about the system to successfully reconstruct the whole phase space with a reasonable choice of $m$. Second, $\tau$ should not be larger than the typical time in which the system loses memory of its initial state. If $\tau$ were chosen larger, the reconstructed phase space would look more or less random since it would consist of uncorrelated points. The latter condition is particularly important for chaotic systems which are intrinsically unpredictable and hence lose memory of the initial state as time progresses. Since the mutual information between $x_{i}$ and $x_{i+\tau}$ quantifies the amount of information we have about the state $x_{i+\tau}$ presuming we know $x_{i}$ [38], Fraser and Swinney [26] proposed to use the first minimum of the mutual information as the optimal embedding delay. For more 


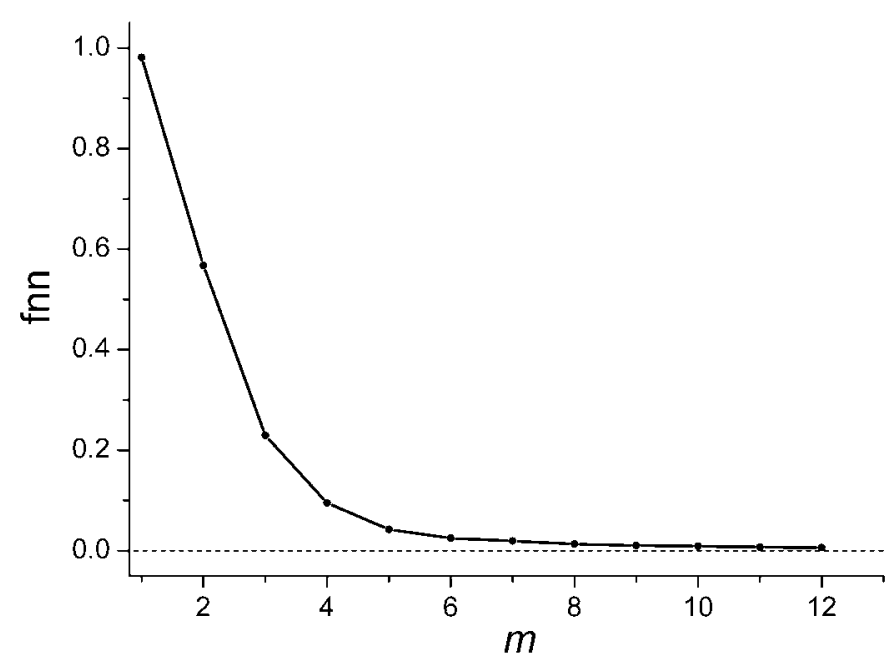

Figure 3. Determination of the minimal required embedding dimension. The fraction of false nearest neighbours (fnn) drops convincingly to zero at $m=10$. This result can be reproduced with the program fnn.exe [36].

interesting aspects on how to choose a proper $\tau$ and the algorithm for calculating the mutual information, we refer the reader to $\mathrm{p} 130$ in [17]. The first minimum of the mutual information calculated for the studied electrocardiographic recording is obtained at $\tau=9$, which is also the value we will use in all subsequent calculations. This result can be reproduced with the program mutual.exe [36].

Let us now turn to establishing a proper embedding dimension $m$ for the examined electrocardiogram by applying the false nearest neighbour method. The false nearest neighbour method was introduced by Kennel et al [27] as an efficient tool for determining the minimal $m$ that is required to fully resolve the deterministic structure of the system in the reconstructed phase space. The method relies on the assumption that the phase space of a deterministic system folds and unfolds smoothly with no sudden irregularities appearing in its structure. By exploiting this assumption, we must come to the conclusion that points that are close in the reconstructed embedding space have to stay sufficiently close during forward iteration also. If a phase space point has a close neighbour that does not fulfil this criterion, it is marked as having a false nearest neighbour. From the geometrical point of view this occurs whenever two points in the phase space solely appear to be close, whereas under forward iteration they are mapped randomly due to projection effects. The random mappings occur because the whole attractor is projected onto a hyperplane that has a smaller dimensionality than the actual phase space and so the distances between points become distorted. As soon as $m$ is chosen sufficiently large, the fraction of points that have a false nearest neighbour (fnn) converges to zero. For the algorithm description we refer the reader to p 39 in [16]. Results obtained with the false nearest neighbour method for $m=1-12$ are presented in figure 3 . It is evident that at least by $m=10$ the fraction of points that have a false nearest neighbour drops convincingly to zero $(<1 \%)$. Hence, the underlying system that produced the studied human electrocardiogram has approximately ten active degrees of freedom. In other words, it would be justified to mathematically model the studied human heart activity with no more than ten first-order ordinary differential equations. Indeed, recent theoretical findings [39] concur with this rather powerful statement. 

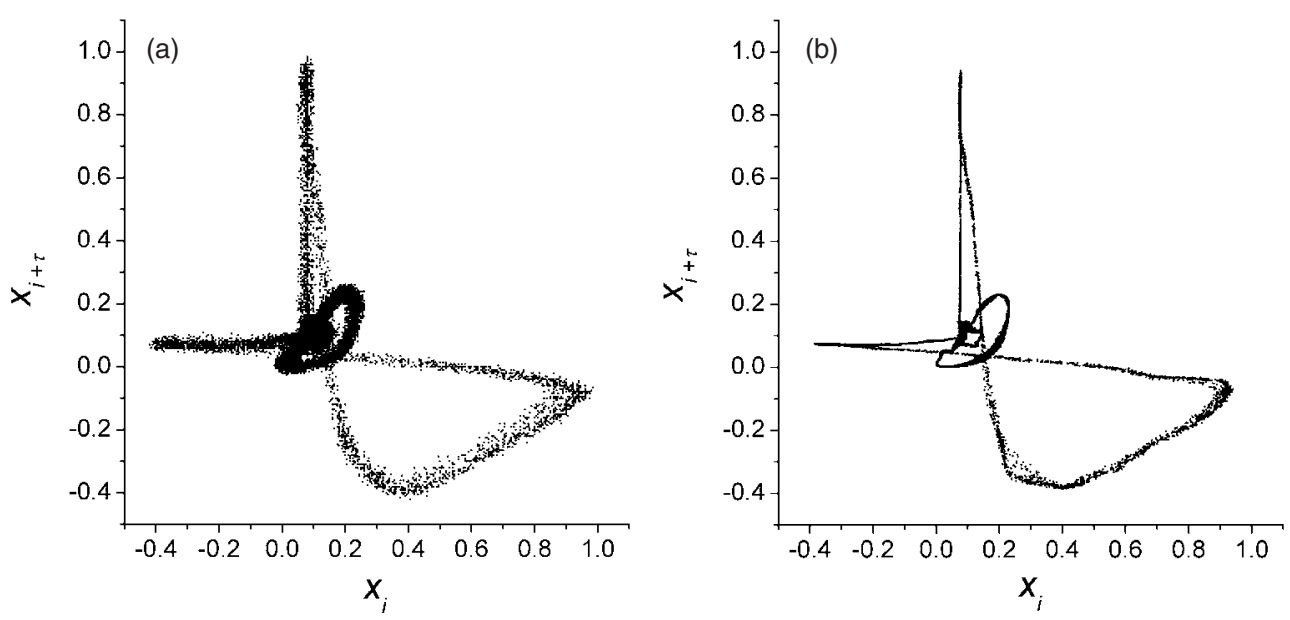

Figure 4. $2 \mathrm{D}$ projection of the reconstructed phase space obtained with the optimal embedding parameters: $\tau=9$ and $m=10$. (a) Before the noise reduction. (b) After the noise reduction. This result can be reproduced with the program embedd.exe [36].

Finally, we have all at hand to present the embedding space of the system obtained according to equation (1). In figure 4 we show 2D projections of the reconstructed phase space for $\tau=9$ and $m=10$. Figure 4(a) was obtained by using the data set before noise reduction, while figure 4(b) shows the result obtained with the 'clean' electrocardiographic signal. It can be observed that the phase space structure in figure 4(b) is much better pronounced than in figure 4(a), which additionally confirms the successfulness of the noise reduction algorithm. Note also that the outline of the phase space was not altered during the noise reduction.

By now we have successfully reconstructed the phase space of the system from a single observed variable. In continuation, it would be possible to apply methods of nonlinear time series analysis that yield invariant quantities of the system, such as for example Lyapunov exponents [40-42] or dimension estimates [43-45], in order to obtain deeper insights into the system dynamics. However, all calculated quantities would be meaningless if the studied time series did not originate from a deterministic stationary system. Thus, in order to justify further analyses, we have to verify if the studied electrocardiogram possesses properties typical of deterministic stationary signals.

An appealing and simple graphical tool that enables the assessment of stationarity and determinism in an observed system is the recurrence plot [30]. Recurrent behaviour is an inherent property of oscillating systems. For regular oscillators time-distinct states in the phase space can be arbitrarily close, i.e. $\|\mathbf{p}(i)-\mathbf{p}(j)\|=0$ if times $i$ and $j$ differ exactly by some integer of the oscillation period, whereas for chaotic systems this distance is always finite. The recurrence plot is a 2D square-grid graph with time units on both axes, whereby, in the most common case [46], points $(i, j)$ that satisfy $\|\mathbf{p}(i)-\mathbf{p}(j)\|<\varepsilon$ are marked with black dots whilst all others are left white. Depending on the application, there also exist several variations of recurrence plots [47, 48], which are however beyond the scope of this paper. The most important feature of each recurrence plot is its large- and small-scale structure, later being termed typology and texture [30], respectively. By visually inspecting the typology and texture of a recurrence plot, properties of the system such as stationarity and determinism can be assessed. In particular, a homogenous typology is an indicator that the studied data set originated from a stationary process. In contrast, a non-homogenous or 
disrupting typology indicates non-stationarity in the system. Texture, on the other hand, can provide information regarding deterministic versus stochastic origin of the signal, as well as give insights into the complexity of oscillations. Lack of texture, i.e. solely isolated recurrence points, often indicates stochastic origin of the examined time series (this is especially true for time-continuous-like recordings as is the studied electrocardiogram), while diagonal lines indicate deterministic oscillations, which depending on the complexity of emerged smallscale patterns can be further classified into simple, complex or chaotic oscillations. The recurrence plot of the studied electrocardiographic signal can be obtained with the program recurrplot.exe [36]. For $\tau=9, m=10, \varepsilon=0.004$ and the 'clean' series as input, the typology is homogeneous whilst the small-scale structure is characterized by diagonal lines of variable length as well as isolated points, indicating the presence of stationary non-trivial deterministic oscillations in the studied electrocardiographic signal. Although, based on a more detailed analysis of the small-scale structure, recurrence plots also enable quantitative assessments of determinism [31] and stationarity [32], as well as other system properties such as Lyapunov exponents, noise level estimates or similarities between two signals [33, 47, 48], we refer the reader to the cited literature for further details and here continue with a classical determinism $[28,49,50]$ and stationarity [29] test to quantify the above-outlined visually assessed results.

We apply a simple yet effective determinism test, originally proposed by Kaplan and Glass [28], that measures average directional vectors in a coarse-grained embedding space. The idea is that neighbouring trajectories in a small portion of the embedding space should all point in the same direction, thus assuring uniqueness of solutions in the phase space, which is the hallmark of determinism. To perform the test, the embedding space has to be coarse grained into equally sized boxes. The average directional vector pertaining to a particular box is obtained as follows. Each pass $p$ of the trajectory through the $k$ th box generates a unit vector $\mathbf{e}_{p}$, whose direction is determined by the phase space point where the trajectory first enters the box and the phase space point where the trajectory leaves the box. In fact, this is the average direction of the trajectory through the box during a particular pass. The average directional vector $\mathbf{V}_{k}$ of the $k$ th box is then simply

$$
\mathbf{V}_{k}=\frac{1}{n} \sum_{p=1}^{n} \mathbf{e}_{p},
$$

where $n$ is the number of all passes through the $k$ th box. Completing this task for all occupied boxes gives us a directional approximation for the vector field of the system. If the time series originates from a deterministic system and the coarse-grained partitioning is fine enough, the obtained directional vector field should consist solely of vectors that have unit length (remember that each $\mathbf{e}_{p}$ is also a unit vector). This follows directly from the fact that we demand uniqueness of solutions in the phase space. If solutions in the phase space are to be unique, then the unit vectors $\mathbf{e}_{p}$ inside each box may not cross, since that would violate the uniqueness condition at each crossing. Note that each crossing decreases the size of the average vector $\mathbf{V}_{k}$. For example, if the crossing of two unit vectors inside the $k$ th box were to occur at right angles, then the size of $\mathbf{V}_{k}$ would be, according to Pythagoras, $\sqrt{2} / 2 \approx 0.707<1$. Hence, if the system is deterministic, the average length of all directional vectors $\kappa$ will be 1 , while for a completely random system $\kappa \approx 0$. The determinism factor pertaining to the ten-dimensional embedding space presented in figure $4(\mathrm{~b})$ that was coarse grained into an $18 \times 18 \times \cdots \times 18$ grid is $\kappa \approx 0.94$, which clearly confirms the deterministic nature of the studied system. On the other hand, the determinism factor pertaining to the embedding space presented in figure $4(\mathrm{a})$ is only $\kappa \approx 0.78$. This result further confirms the successfulness of the noise reduction scheme [25], but also stresses the necessity for its application. The above results can be reproduced with the program determinism.exe [36]. 


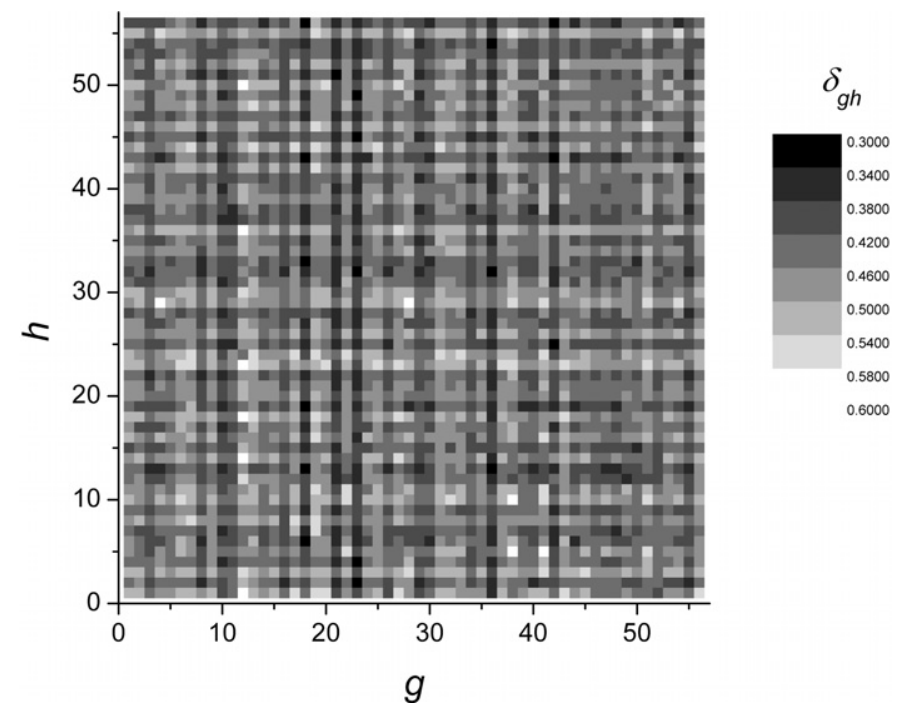

Figure 5. Stationarity test. The whole time series was partitioned into 56 non-overlapping segments each occupying 800 data points. The colour map displays average cross-prediction errors $\delta_{g h}$ in dependence on different segment combinations. This result can be reproduced with the program stationarity.exe [36].

It remains of interest to verify if the studied electrocardiographic signal indeed originated from a stationary process, as indicated by the recurrence plot analysis. To this purpose, we apply the stationarity test proposed by Schreiber [29]. In general, stationarity violations manifest so that various non-overlapping segments of the time series have different dynamical properties. Since linear statistics, such as the mean or standard data deviation, usually do not possess enough discrimination power when analysing irregular signals, nonlinear statistics have to be applied. One of the most effective has proven to be the cross-prediction error statistic. The idea is to split the time series into several short non-overlapping segments and then use a particular data segment to make predictions in another data segment. This task then has to be repeated for all possible combinations. For example, if we divide the original data set into short series each occupying 800 points, we then have a total of 56 segments and exactly $56^{2}$ possible combinations to evaluate the statistics. By calculating the average prediction error $\left(\delta_{g h}\right)$ when considering points in segment $g$ to make predictions in segment $h$, we obtain a very sensitive statistic capable of detecting minute changes in dynamics and thus a very powerful probe for stationarity. If for any combination of $g$ and $h, \delta_{g h}$ is significantly above average, this is a clear indicator that the examined data set originated from a non-stationary process. An accurate description of the whole algorithm can be found in [17] from p 42 onwards, while here we concentrate on the results. The average cross-prediction errors for all possible combinations of $g$ and $h$ are presented in figure 5. The average value of all $\delta_{g h}$ is 0.45 , while the minimum and maximum values are 0.32 and 0.60 , respectively. Since the maximal cross-prediction error is not even one time larger than the average and all cross-prediction errors differ maximally by a factor of 2 , we can clearly refute non-stationarity in the studied time series. We emphasize, however, that stationarity can be positively established solely because we consider only $180 \mathrm{~s}$ of the electrocardiographic recording. Otherwise, longer recordings of physiological functions almost always yield non-stationary data sets.

Above, we have advocated a very careful approach, which we believe should always be followed before attempting further analyses. It is the best protection against spurious 


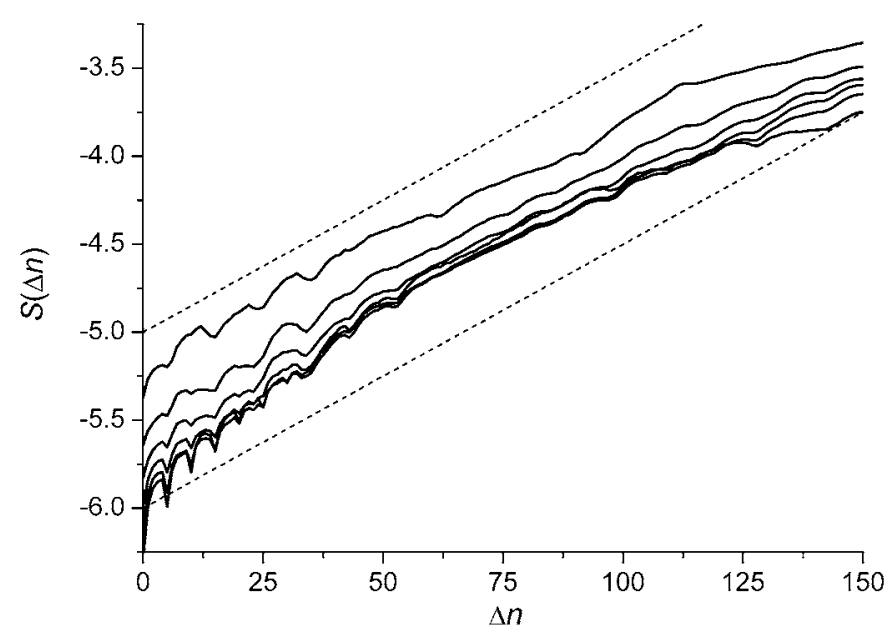

Figure 6. Calculation of the maximal Lyapunov exponent. The slope of the linear part of the graph indicated by the two dashed lines is a good estimate for the maximal Lyapunov exponent. This result can be reproduced with the program lyapmaxk.exe [36].

results, and above all, is far superior than any level of sophistication one might achieve in applied methods, meaning that even the best algorithms would not prevent one from obtaining wrong and meaningless results if applied to data sets that largely lack determinism and/or stationarity. As a reward, we can now proceed and calculate the maximal Lyapunov exponent without having to worry about the origin and spuriousness of the obtained value.

In order to calculate the maximal Lyapunov exponent of the system, we use the algorithm developed independently by Kantz [35] and Rosenstein et al [51]. The algorithm tests the exponential divergence of nearby trajectories directly and thus allows a robust estimation of the maximal Lyapunov exponent. To estimate the exponent, we first have to find all neighbours $\mathbf{p}(k)$ that are closer to a particular reference point $\mathbf{p}(i)$ than $\varepsilon$. Thereby, we obtain a set of starting points for nearby trajectories. Further, we have to calculate the average distance of all trajectories to the reference trajectory $\left(D_{i}\right)$ as a function of the relative time $\Delta n$ (counted from $i$ and $k$ onwards). Finally, the average of the logarithm of $D_{i}$, obtained for several different reference points $\mathbf{p}(i)$, is the effective expansion rate $S(\Delta n)$, of which the linear slope in dependence on $\Delta n$ is a robust estimate for the maximal Lyapunov exponent. To obtain an accurate result, the whole algorithm has to be repeated for a few hundred different $\mathbf{p}(i)$ and various $\varepsilon$. In particular, $\varepsilon$ should be chosen as small as possible, but still large enough so that on average each reference point $\mathbf{p}(i)$ has at least a few neighbours. For further details we refer the reader to the original articles [35, 51] and also to p 62 in [17]. The results obtained for $\varepsilon=0.01-0.056$ are presented in figure 6. The function $S(\Delta n)$ shows a rather robust linear increase from $\Delta n=25$ to 125 for all $\varepsilon$. Thus, the slope of $S(\Delta n)$, indicated by the two linear lines, is a good estimate for the maximal Lyapunov exponent of the system. We find that the latter equals $\approx 0.015$, from which we conclude that the studied short stationary data segment of the human electrocardiogram possesses properties typical of deterministic chaotic signals.

\section{Discussion}

We systematically analyse the human electrocardiogram with basic methods of nonlinear time series analysis. In particular, we outline a careful approach that largely eliminates the 
occurrence of spurious results and thus guarantees a relevant analysis of the observed system. We find that short, densely sampled electrocardiographic recordings may show properties that are typical of deterministic chaotic systems. Moreover, to facilitate interest and enable the integration of nonlinear time series analysis methods into the curriculum at an early stage of the educational process, we provide user-friendly programs with graphical interface for each implemented method [36]. Hopefully, this will make the reproduction of presented results possible even for individuals with little or no experience with nonlinear time series analysis and inspire them to get familiar with the presented theory.

Although our findings concur with results published in recent studies [52], it should be noted that in general the origin of observed irregular behaviour in the human cardiovascular system is a subject of some debate [52-55]. Especially, time series describing heart rate variability that are derived from long continuous electrocardiographic recordings often lack determinism and stationarity. Thus, we emphasize that the above study is intended as a comprehensive introduction to basic nonlinear time series analysis of physiological data, but should not be considered as the definite resource for characterization of the dynamics of the human heart. To obtain a well-balanced view on issues concerning the presence of determinism and chaos in the human cardiovascular system, we strongly advise the reader to consult [52-55] for additional information.

Finally, we note that the methods described in this paper represent an excellent starting point for more advanced studies, such as wavelet analyses to determine couplings in the human cardio-respiratory system [56] or applications of nonlinear state-space projections to extract the foetal ECG from the maternal electrocardiographic recordings [57]. Currently, these topics are covered in existing monographs on nonlinear time series analysis [16-18] and in the original papers. An excellent source of information for these methods is also the TISEAN project [58]. Together with the pertaining paper [59] and the book by the same authors [17], the TISEAN project presents a very valuable source of information as well as programs for virtually all topics of nonlinear time series analysis. Therefore, particularly for students that would like to delve deeper into the theory of nonlinear time series analysis, we recommend to exploit the benefits offered by these sources.

\section{References}

[1] Lorenz E N 1963 Deterministic nonperiodic flow J. Atmos. Sci. 20 130-41

[2] MacDonald N and Whitehead R R 1985 Introducing students to nonlinearity: computer experiments with Burgers mappings Eur. J. Phys. 6 143-7

[3] Nunez Yepez H N, Salas Brito A L, Vargas C A and Vicente L A 1989 Chaos in a dripping faucet Eur. J. Phys. 10 99-105

[4] Rodgers G J 1992 From order into chaos Phys. Educ. 27 14-7

[5] Carretero-Gonzalez R, Nunez-Yepez H N and Salas-Brito A L 1994 Regular and chaotic behaviour in an extensible pendulum Eur. J. Phys. 15 139-48

[6] Barrientos M, Perez A and Ranada A F 1995 Weak chaos in the asymmetric heavy top Eur. J. Phys. 16 106-12

[7] Borcherds P H 1995 The butterfly that stamped: a brief introduction to nonlinear dynamics and chaos Phys. Educ. 30 372-81

[8] Hobson P R and Lansbury A N 1996 A simple electronic circuit to demonstrate bifurcation and chaos Phys. Educ. 31 39-43

[9] Schmidt T and Marhl M 1997 A simple mathematical model of a dripping tap Eur. J. Phys. 18 377-83

[10] Martin S J and Ford P J 2001 A simple experimental demonstration of chaos in a driven spherical pendulum Phys. Educ. 36 108-14

[11] Gitterman M 2002 Order and chaos: are they contradictory or complementary? Eur. J. Phys. 23 119-22

[12] Tamaševičius A, Mykolaitis, Pyragas V and Pyragas K 2005 A simple chaotic oscillator for educational purposes Eur. J. Phys. 26 61-3

[13] Perc M 2005 The dynamics of human gait Eur. J. Phys. 26 525-34

[14] Perc M 2005 Visualizing the attraction of strange attractors Eur. J. Phys. 26579

[15] Kodba S, Perc M and Marhl M 2005 Detecting chaos from a time series Eur. J. Phys. 26 205-15 
[16] Abarbanel H D I 1996 Analysis of Observed Chaotic Data (New York: Springer)

[17] Kantz H and Schreiber T 1997 Nonlinear Time Series Analysis (Cambridge: Cambridge University Press)

[18] Sprott J C 2003 Chaos and Time-Series Analysis (Oxford: Oxford University Press)

[19] Schuster H G 1989 Deterministic Chaos (Weinheim: VCH)

[20] Ott E 1993 Chaos in Dynamical Systems (Cambridge: Cambridge University Press)

[21] Strogatz S H 1994 Nonlinear Dynamics and Chaos (Reading, MA: Addison-Wesley)

[22] Kaplan D T and Glass L 1995 Understanding Nonlinear Dynamics (New York: Springer)

[23] Takens F 1981 Detecting Strange Attractors in Turbulence (Lecture Notes in Mathematics vol 898) ed D A Rand and L S Young (Berlin: Springer) p 366

[24] Sauer T, Yorke J A and Casdagli M 1991 Embedology J. Stat. Phys 65 579-616

[25] Schreiber T 1993 Extremely simple nonlinear noise reduction method Phys. Rev. E 47 2401-4

[26] Fraser A M and Swinney H L 1986 Independent coordinates for strange attractors from mutual information Phys. Rev. A $331134-40$

[27] Kennel M B, Brown R and Abarbanel H D I 1992 Determining embedding dimension for phase space reconstruction using a geometrical construction Phys. Rev. A 45 3403-11

[28] Kaplan D T and Glass L 1992 Direct test for determinism in a time series Phys. Rev. Lett. 68 427-30

[29] Schreiber T 1997 Detecting and analyzing nonstationarity in a time series with nonlinear cross-predictions $P$ hys. Rev. Lett. 78 843-6

[30] Eckmann J-P, Kamphorst S O and Ruelle D 1987 Recurrence plots of dynamical systems. Europhys. Lett. 5 973-7

[31] Atay F M and Altintas Y 1999 Recovering smooth dynamics from time series with the aid of recurrence plots Phys. Rev. E 59 6593-8

[32] Manuca R and Savit R 1996 Stationarity and nonstationarity in time series analysis Physica D 99 134-61

[33] Urbanowicz K and Holyst J A 2003 Noise-level estimation of time series using coarse-grained entropy Phys. Rev. E 67046218

[34] Zbilut J P and Webber C L Jr 1992 Embeddings and delays as derived from quantification of recurrence plots Phys. Lett. A 171 199-203

[35] Kantz H 1994 A robust method to estimate the maximal Lyapunov exponent of a time series Phys. Lett. A 185 77-87

[36] All results presented in this paper can be easily reproduced with the set of programs that can be downloaded from http://fizika.uni-mb.si/ matjaz/ejp/time.html

[37] Goldberger A L, Amaral L A N, Glass L, Hausdorff J M, Ivanov P Ch, Mark R G, Mietus J E, Moody G B, Peng C K and Stanley H E 2000 PhysioBank, PhysioToolkit, and PhysioNet: components of a new research resource for complex physiologic signals Circulation 101 e215-20 (Circulation Electronic Pages: http://circ.ahajournals.org/cgi/content/full/101/23/e215)

[38] Shaw R 1981 Strange attractors, chaotic behavior, and information flow Z. Naturforsch. 36a 80-112

[39] McClintock P V E and Stefanovska A 2002 Noise and determinism in cardiovascular dynamics Physica A 314 69-76

[40] Briggs K 1990 An improved method for estimating Lyapunov exponents of chaotic time series Phys. Lett. A $15127-32$

[41] Abarbanel H D I, Brown R and Kennel M B 1992 Local Lyapunov exponents from observed data J. Nonlinear Sci 2 343-65

[42] Parlitz U 1992 Identification of true and spurious Lyapunov exponents from time series Int. J. Bifurcat. Chaos 2 155-65

[43] Grassberger P and Procaccia I 1983 Characterization of strange attractors Phys. Rev. Lett. 50 346-9

[44] Theiler J 1986 Spurious dimension from correlation algorithms applied to limited time-series data Phys. Rev. A 34 2427-32

[45] Kantz H and Schreiber T 1994 Dimension estimates and physiological data Chaos 5 143-54

[46] Zbilut J P, Koebbe M, Loeb H and Mayer-Kress G 1991 Use of recurrence plots in the analysis of heart beat intervals Proc. IEEE Conf. Computers in Cardiology (Chicago, 1990) (Los Alamitos, CA: IEEE Computer Society Press) pp 263-6

[47] Marwan N 2003 Encounters with neighbours-current developments of concepts based on recurrence plots and their applications $P h D$ Thesis University of Potsdam

[48] Marwan N 2003 Recurrence Plot Web site http://www.recurrence-plot.tk

[49] Wayland R, Bromley D, Pickett D and Passamante A 1993 Recognizing determinism in a time series Phys. Rev. Lett. 70 580-2

[50] Salvino L W and Cawley R 1994 Smoothness implies determinism: a method to detect it in time series Phys. Rev. Lett. 73 1091-4

[51] Rosenstein M T, Collins J J and De Luca C J 1993 A practical method for calculating largest Lyapunov exponents from small data sets Physica D 65 117-34

[52] Kantz H and Schreiber T 2002 The human ECG-nonlinear deterministic versus stochastic aspects IEE Proc. Sci. Meas. Technol. 145 279-84

[53] Kaplan D T and Cohen R J 1990 Is fibrillation chaos? Circ. Res. 67 886-92

[54] Goldberger A L 1991 Is the normal heartbeat chaotic or homeostatic? News Physiol. Sci. 6 87-91 
[55] Kanters J K, Holstein-Rathlou N-H and Agner E 1994 Lack of evidence for low-dimensional chaos in heart rate variability J. Cardiovasc. Electrophysiol 5 591-601

[56] Bračič M and Stefanovska A 1998 Wavelet based analysis of the human blood flow dynamics Bull. Math. Biol. 60 919-35

[57] Richter M, Schreiber T and Kaplan D T 1998 Fetal ECG extraction with nonlinear state-space projections IEEE Trans. Biomed. Eng. $45133-7$

[58] The official web page of the TISEAN project is www.mpipks-dresden.mpg.de/ tisean

[59] Hegger R and Kantz H 1999 Practical implementation of nonlinear time series methods: the TISEAN package Chaos 9 413-40 